\title{
Peripheral refraction with eye and head rotation with contact lenses
}

\author{
Daniela P. Lopes-Ferreira, Helena I.F. Neves, Miguel Faria-Ribeiro, António Queirós, \\ Paulo R.B. Fernandes, José M. González-Méijome*
}

Clinical and Experimental Optometry Research Laboratory (CEORLab), Center of Physics (Optometry), School of Sciences, University of Minho, Braga, Portugal

\section{A R T I C L E I N F O}

\section{Article history:}

Received 27 October 2013

Received in revised form

19 November 2014

Accepted 24 November 2014

\section{Keywords:}

Peripheral refraction

Myopia

Multifocal contact lens

Eye rotation

Head rotation

\begin{abstract}
A B S T R A C T
Purpose: To evaluate the impact of eye and head rotation in the measurement of peripheral refraction with an open-field autorefractometer in myopic eyes wearing two different center-distance designs of multifocal contact lenses (MFCLs).

Methods: Nineteen right eyes from 19 myopic patients (average central $\mathrm{M} \pm \mathrm{SD}=-2.67 \pm 1.66 \mathrm{D}$ ) aged 20-27 years (mean $\pm \mathrm{SD}=23.2 \pm 3.3$ years) were evaluated using a Grand-Seiko autorefractometer. Patients were fitted with one multifocal aspheric center-distance contact lens (Biofinity Multifocal $D^{\circledR}$ ) and with one multi-concentric MFCL (Acuvue Oasys for Presbyopia). Axial and peripheral refraction were evaluated by eye rotation and by head rotation under naked eye condition and with each MFCL fitted randomly and in independent sessions.

Results: For the naked eye, refractive pattern (M, J0 and J45) across the central $60^{\circ}$ of the horizontal visual field values did not show significant changes measured by rotating the eye or rotating the head $(p>0.05)$. Similar results were obtained wearing the Biofinity $D$, for both testing methods, no obtaining significant differences to M, J0 and J45 values ( $p>0.05)$. For Acuvue Oasys for presbyopia, also no differences were found when comparing measurements obtained by eye and head rotation $(p>0.05)$. Multivariate analysis did not showed a significant interaction between testing method and lens type neither with measuring locations (MANOVA, $p>0.05$ ). There were significant differences in M and J0 values between naked eyes and each MFCL.

Conclusion: Measurements of peripheral refraction by rotating the eye or rotating the head in myopic patients wearing dominant design or multi-concentric multifocal silicone hydrogel contact lens are comparable.
\end{abstract}

(C) 2014 Published by Elsevier Ltd on behalf of British Contact Lens Association.

\section{Introduction}

Peripheral refraction has been studied extensively since it was suggested that it might play a role in the refractive development of the eye, particularly, in myopia progression [1,2]. Researchers have observed that the peripheral refraction was relatively more hyperopic in myopic eyes than in emmetropic eyes along the horizontal visual field [3]. There are also differences in the peripheral refraction and retinal contour between progressing and stable myopes [4]. A previous animal study reported that peripheral hyperopic defocus (behind the retina) could induce central myopic development [5].

\footnotetext{
* Corresponding author at: Clinical and Experimental Optometry Research Laboratory (CEORLab), Department of Physics (Optometry), University of Minho, 4710-057 Braga, Portugal. Tel.: +351 25360 4320; fax: +35125367 8981 .

E-mail address: jgmeijome@fisica.uminho.pt (J.M. González-Méijome).
}

Myopia correction with conventional spectacles may increase relative peripheral hyperopic defocus [6,7], especially in high degrees of myopia and at larger eccentricities of the visual field [8]. Considering the evidence that orthokeratology slows myopia progression [9-14] and that this treatment induces a substantial change in the peripheral refractive error [15] of the myopic eye toward high degrees of peripheral myopic defocus and astigmatism, a link has been suggested between relative peripheral hyperopic defocus and myopia progression in humans [9,11]. Some ophthalmic lenses [7] and contact lenses [16] have been designed specifically to arrest myopia progression based on this hypothetical mechanism. The main goal of the commercially available centerdistance design multifocal contact lenses (MFCLs) is to compensate for presbyopia. However, considering the similar change in the peripheral refractive pattern induced by these lenses [17-19], it has been hypothesized that such designs can be useful to slow myopia progression [20]. Bifocal contact lenses for presbyopia have previously been used to slow myopia progression [21,22]. Recently, a 
dual-focus contact lens has been proved to be effective in reducing myopia progression by up to $34 \%$ in children over a 10 -month period [23]. Kollbaum et al. recently evaluated the quality of vision of center-distance design and bifocal contact lenses for presbyopia and compared them to dual-focus lenses to determine the potential use of such lenses to control myopia [24]. Although not all of these devices are intended to induce peripheral myopic defocus, it might be of interest to evaluate the potential contribution of this factor with each lens design. However, when evaluating the potential of different multifocal devices for changing the peripheral myopic refractive pattern with contact lenses on the eye, ocular and head rotation might be a concern. Seidemann et al. [25] hypothesized that pressure exerted by the extraocular muscles and the eyelids on eye rotation might distort the shape of the eyeball and alter refraction across the visual field. However, Radhakrishnan and Charman reported that for the naked eye this might not be relevant [26]. This might be potentially different with a contact lens in place considering the effect of decentration during peripheral gaze. However, this effect remains controversial, and several authors have preferred to measure the peripheral refraction by rotating the head $[16,27,28]$, while others performed such measurements with eye rotation $[29,30]$.

The current study was conducted to evaluate the effect of ocular and head rotation on the peripheral refraction measurements obtained with an open-field autorefractor in myopic eyes using two different center-distance designs of MFCLs comprising an aspheric multifocal design and a concentric multifocal design.

\section{Methods}

The experiments were conducted at the Clinical and Experimental Optometry Research Lab (CEORLab, Minho University, Braga, Portugal). All participants were fully informed about the purpose and procedures of this study and provided written consent. The study followed the tenets of the Declaration of Helsinki; the Scientific Committee of the School of Sciences of Minho University (Portugal) approved the research protocol.

Nineteen healthy young subjects were recruited from a university population. Inclusion criteria required that patients had 20/20 monocular visual acuity, myopia lower than -8.00 diopters (D), astigmatism lower than $-1.00 \mathrm{D}$ as measured by subjective refraction, no ocular disease or injury, no history of refractive surgery, and no use of ocular or systemic medication.

\subsection{MFCLs}

The right eyes of the participants were fitted randomly in independent sessions with two MFCLs that included a distance vision zone with their foveal refractive correction. The Biofinity ${ }^{\circledR}$ Multifocal D (Comfilcon A, Coopervision, Pleasanton, CA, USA) is a new multifocal contact lens with an optical design and fitting procedure similar to those of the Proclear Multifocal D (Omafilcon A, Coopervision, Pleasanton, CA, USA). The Biofinity D lens has an aspheric center-distance multifocal design with more positive power in the outer zone of the lens. The optical design consists of a spherical central zone of $2.3 \mathrm{~mm}$ in diameter dedicated to distance vision, surrounded by an annular aspheric zone of $5.0 \mathrm{~mm}(1.35 \mathrm{~mm}$ width) of increasing addition power and a spherical annular zone of $8.5 \mathrm{~mm}$ ( $1.50 \mathrm{~mm}$ width) reaching the maximum add power. The second lens, the Acuvue ${ }^{\circledR}$ Oasys for Presbyopia (Senofilcon A, Johnson \& Johnson, Jacksonville, FL, USA), has a multi-concentric design with center-distance area of about $2.0 \mathrm{~mm}$ followed by multiple alternating near and distance concentric zones (between 0.5 and $1.0 \mathrm{~mm}$ width) from the center to the end of the optical zone at $8.0 \mathrm{~mm}$. The maximal add power in both MFCLs was $+2.50 \mathrm{D}$ to guarantee that equivalent add powers were available in both lenses, and the add power was closer to the one that yielded the best peripheral myopic defocus effect with the Proclear D lens in our previous study [19]. After a previous fitting session during which the optimum centration (less than $0.5 \mathrm{~mm}$ of lateral displacement against the limbal area) and movement (lag $<0.5 \mathrm{~mm}$ on lateral and upgaze) were assessed.

\subsection{Central and peripheral refraction}

The non-cycloplegic objective refraction was obtained in the right eye using an open-field autorefractor/keratometer GrandSeiko WAM-5500 (Grand Seiko Co., Ltd., Hiroshima, Japan) previously used to measure the central and peripheral refractions $[31,32]$. The illumination in the examination room was adjusted to obtain sufficiently large pupils to facilitate peripheral measurements. The central and peripheral non-cycloplegic refractions were evaluated for the naked eye and with both MFCLs. The left eye was always occluded during measurements. Head and eye rotation measurements were performed randomly during the same session 5 min apart from each other.

The peripheral refraction was obtained using an array of lightemitting diodes (LEDs) with a diameter of $5 \mathrm{~mm}$ located at $2.5 \mathrm{~m}$ along the horizontal visual field at eccentricities between $30^{\circ}$ nasally and $30^{\circ}$ temporally, in $10^{\circ}$ steps. For the eye rotation measurements, the patient was instructed to fixate on the LEDs as previously described [26,33]. For the head rotation measurements, we used a previously reported method $[28,26]$, during which a laser pointer positioned on top of the patient's head was oriented toward the primary gaze position. Room light was kept at low intensity (about $20 \mathrm{~cd} / \mathrm{m}^{2}$, low photopic level) in order to avoid pupil miosis. Under these conditions, the pupil size was large enough to allow measures to be obtained through the elliptical pupil when the eye or head rotated. The patient rotated his or her head, avoiding lateral displacement, until the pointer reached the desired eccentric LED while the eyes remained in the primary gaze position. Individual data were transposed into vector components according to Fourier analysis [34,35]. Five refractive measurements were performed and averaged after transposition into the vector components ( $\mathrm{M}, \mathrm{J} 0$ and J45) for each eccentricity. The refractive data were saved automatically in Microsoft Excel spreadsheets using custom software (DRRE, CEORLab).

\subsection{Statistical analysis}

The data were analyzed using SPSS for Windows, version 20 (SPSS Inc., New York, USA). The Shapiro-Wilk test was applied to evaluate the normality of the data distribution. Relative peripheral refractive error was obtained by subtracting the central refractive error to the refractive component ( $\mathrm{M}, \mathrm{J} 0$ or J45) at each eccentric location $\left(10,20,30^{\circ}\right.$ nasal or temporal). The behaviors between the relative peripheral refractive patterns between ocular and head rotation were evaluated for each condition (naked eye, Acuvue Oasys lens for presbyopia, and Biofinity Multifocal D lens). The effect of factors such as measurement location (eccentricities), lens type (naked eye, Acuvue Oasys lens for presbyopia, or Biofinity D lens), and testing method (eye or head rotation) on the mean values of the dependent variables (MJ0, and J45) were evaluated using multivariate analysis of variance (MANOVA). When MANOVA detected the statistically significant effects $(p<0.05)$ of a certain factor, we performed an individual ANOVA for each dependent variable, followed by the Bonferroni post hoc test. A $p$ value lower than 0.05 was considered statistically significant. 

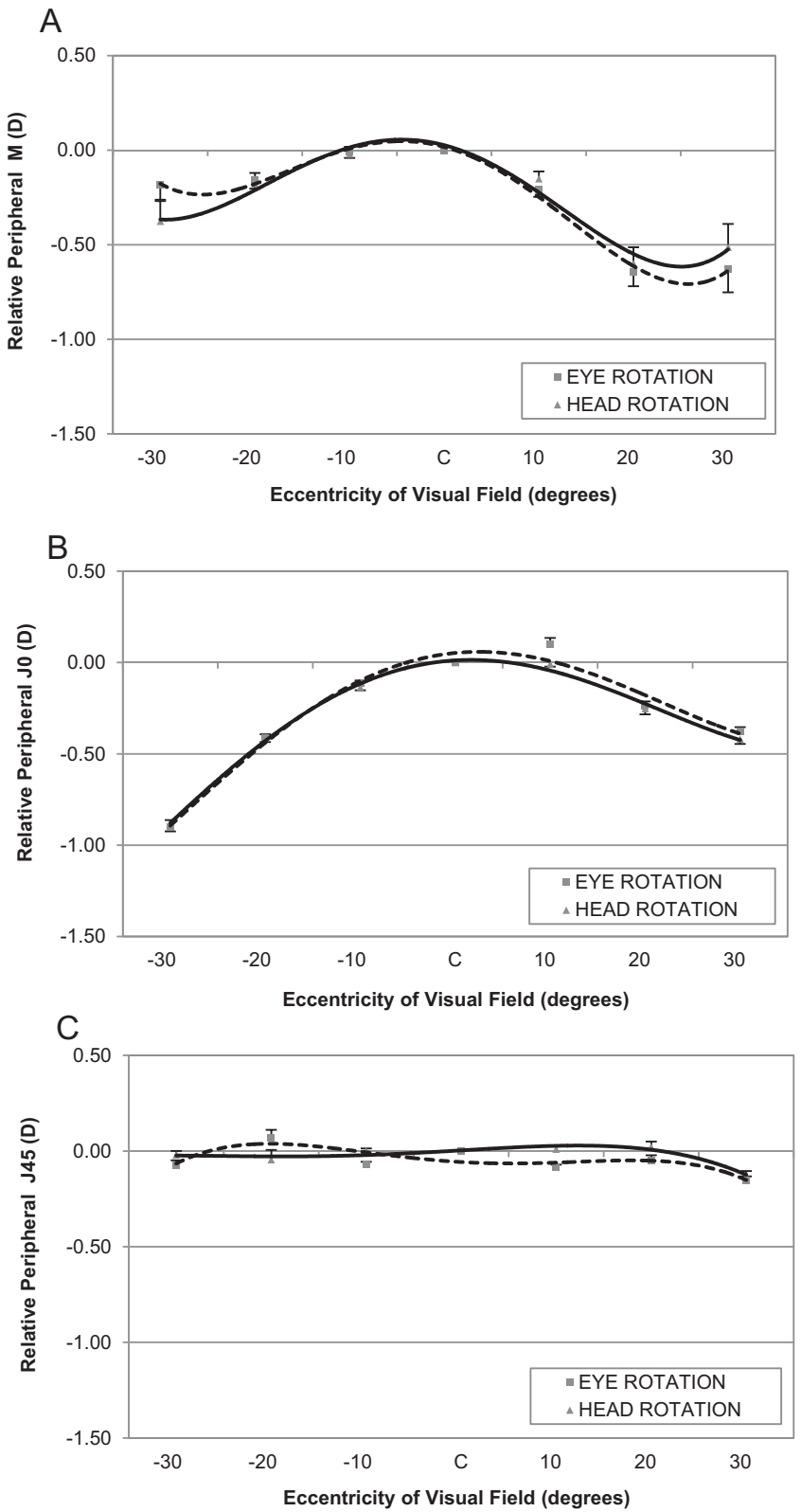

Fig. 1. Relative peripheral refractive error measured in naked eyes (eccentric location minus center) by eye rotation (squares, dashed line) and head rotation (triangles, solid line). (A) Spherical equivalent values (M); (B) horizontal component of astigmatism (J0) and (C) oblique component of astigmatism (J45) across the central $60^{\circ}$ of the horizontal visual field (nasal visual field eccentricities as positive). The error bars represent the standard error of the mean.

\section{Results}

We evaluated right eyes of 19 young students (13 women, 6 men) (mean age \pm standard deviation [SD], $23.2 \pm 3.3$ years), mean central refractive error $(\mathrm{M} \pm \mathrm{SD})$ of $-2.67 \pm 1.66 \mathrm{D}$.

Fig. $1 \mathrm{~A}-\mathrm{C}$ shows the average relative peripheral (eccentric values minus central value) values of $\mathrm{M}$, J0, and J45 obtained by testing methods evaluated (eye and head rotation) in naked eyes. The $\mathrm{M}$ component showed greater differences at $30^{\circ}$ nasally $(-0.12 \pm 0.30 \mathrm{D} ; p<0.001)$ and $30^{\circ}$ temporally $(0.19 \pm 0.59 \mathrm{D}$; $p=0.002$ ), but neither achieved statistical significance. Fig. 2A-C shows the comparison of the peripheral refractive errors between testing methods in eye fitted with the Acuvue Oasys lens for presbyopia. The values obtained by rotating the eye were slightly more myopic than those obtained with head rotation but neither
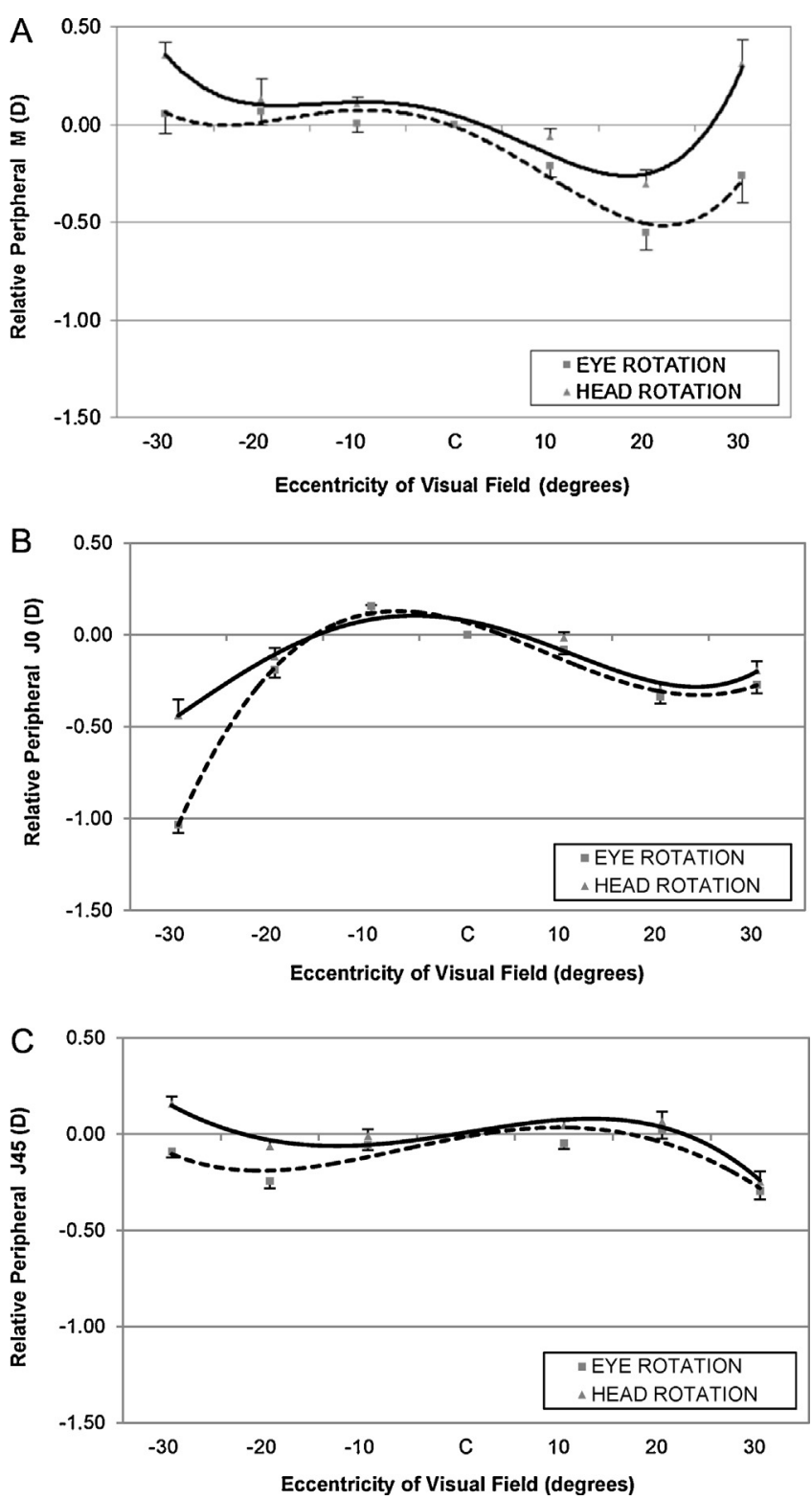

Fig. 2. Relative peripheral refractive errors measured with Acuvue Oasys for presbyopia (eccentric location minus center) by rotating the eye (squares, dashed line) and rotating the head (triangles, solid line). (A) Spherical equivalent values (M); (B) horizontal component of astigmatism (J0) and (C) oblique component of astigmatism (J45) across the central $60^{\circ}$ of the horizontal visual field (nasal visual field eccentricities as positive). The error bars represent the standard error of the mean.

statistically difference were found to M values $(p=0.761)$. Similar results were found with the J0 and J45 component; measuring by eye rotation the values manifested more myopia for all eccentricities, however differences did not reach to the statistical significance ( $p=0.050$ and $p=0.479$, respectively).

Finally, the peripheral refractive changes induced by the Biofinity Multifocal D lens are shown in Fig. 3A-C. The relative peripheral $M$ values were similar between the two testing methods used, no statistically differences were found $(p=0.345)$. The J0 component was more myopic at the more eccentric temporal points $\left(20\right.$ and $30^{\circ}$ ) measured by head rotation, and the J45 component showed lower hyperopic values by eye rotation method. However, differences between head and eye rotation were not statistically significant for both astigmatic components $(p=0.777$ and $p=0.255$, respectively). 


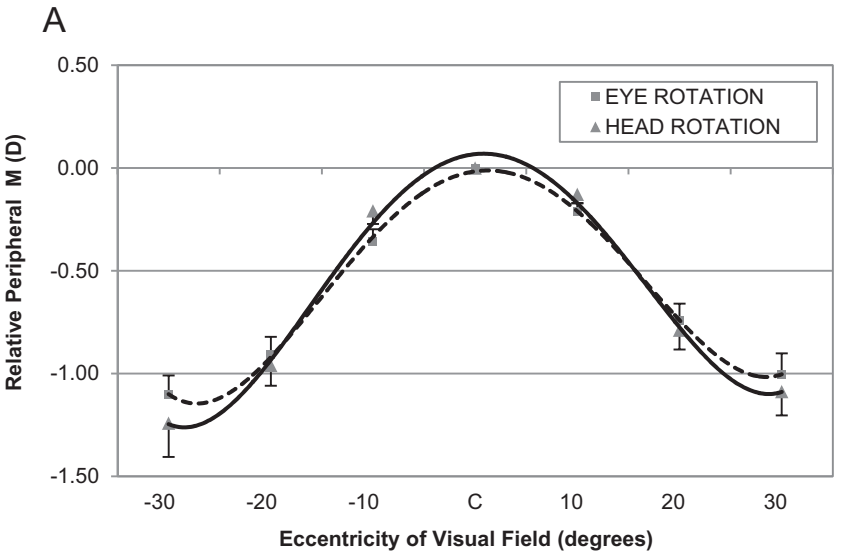

B
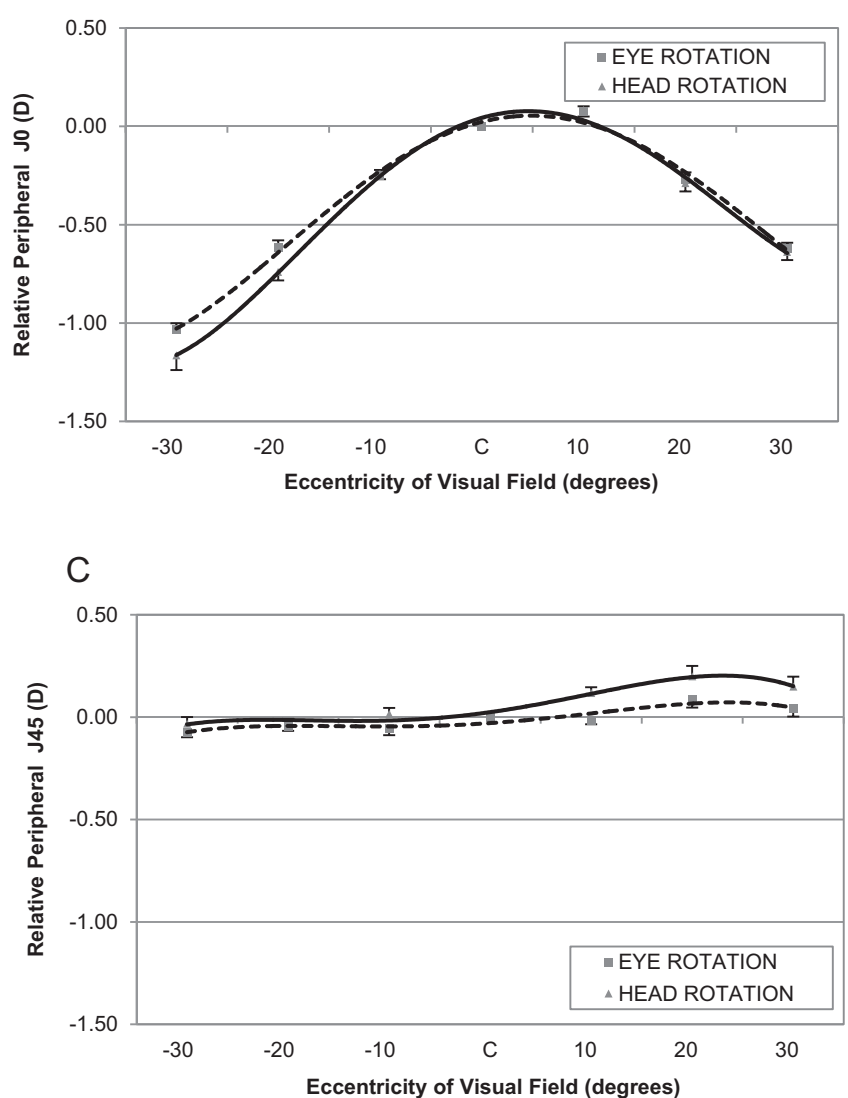

Fig. 3. Relative peripheral refractive errors measured with Biofinity Multifocal D design (eccentric location minus center) by rotating the eye (squares, dashed line) and rotating the head (triangles, solid line). (A) Spherical equivalent values (M); (B) horizontal component of astigmatism (J0) and (C) oblique component of astigmatism (J45) across the central $60^{\circ}$ of the horizontal visual field (nasal visual field eccentricities as positive). The error bars represent the standard error of the mean.

No significant interactions were found between the testing method and location or between the testing method and lens type for M, J0, and J45 ( $p>0.05$ in all comparisons by MANOVA analysis). Significant interaction was observed between location and lens type ( $p<0.001$, MANOVA analysis). Between-subjects effects also showed that this interaction was significant for all dependent variables $\mathrm{M}$, J0, and $\mathrm{J} 45$ ( $p<0.005$ for all comparisons). After adjusting for multiple comparisons analysis followed by ANOVA analysis and Bonferroni post hoc test, the results indicated that peripheral relative $M$ was significantly different between naked eyes and the same eyes fitted with each MFCL; the differences were significant for all point-to-point comparisons between the naked eye and the Acuvue Oasys lens for presbyopia ( $p<0.001$, ANOVA with the Bonferroni post hoc test) and the naked eye and the Biofinity D Multifocal lens, being less myopic with the naked eyes; statistically less myopic in all points in case of first comparison and except for 20 and $30^{\circ}$ temporally for the second $(p=0.092$ and $p=0.840$ respectively). However, in the comparisons of the two MFCLs studied, the M values did not differ significantly ( $p=0.969$ ). Peripheral relative J0 values also differed significantly between the naked eyes and the MFCLs $(p<0.001)$, and between the lens Acuvue Oasys for presbyopia and the Biofinity $\mathrm{D}(p<0.001)$. In contrast, J45 did not show significant differences between naked eyes and the MFCL conditions or between the MFCLs $(p=0.076)$.

\section{Discussion}

The current study aimed to investigate if peripheral refraction (PR) measurements using an open-field autorefractor were different between head rotation and eye rotation in myopic eyes while they were without lenses or wearing 2 different brands of MFCLs. Comparisons between the eye rotation and head rotation methods in the assessment of peripheral refraction [26] and ocular shape [36] have already been performed but only for naked eyes. According to the current results and independent of the method that was performed, the peripheral refractive pattern was similar in the naked eye as Radhakrishnan and Charman [26] previously described using an autorefractor and by Mathur et al. [33] using an aberrometer.

Considering the interest in the effectiveness of optical devices, such as MFCLs, to produce relative peripheral myopic defocus or to modulate the accommodative effort of the eye, it is relevant to understand if reliable results can be obtained when measuring peripheral refraction in eyes wearing a soft contact lens. Ocular rotation may induce contact lens displacement by mechanical interaction with the eyelids. Thus, some authors have used the head rotation method in experiments in which patients were fitted with a contact lens [27,28]. However, recently Kang et al. [30] measured the peripheral refraction with a contact lens by rotation of the eye to evaluate the effect of under-correction, full-correction, and overcorrection in the peripheral refraction of young subjects. Ticak and Walline also measured the peripheral refraction with Proclear Multifocal D Soft Contact Lenses by rotating the eye [29]. The current results supported this approach, in that we did not find significant differences in $\mathrm{M}, \mathrm{J} 0$, or $\mathrm{J} 45$ obtained by rotating the eye or the head. This might be supported by the small movement of modern soft contact lenses on the average eye. Young et al. [37] previously estimated the lag of soft contact lenses on upgaze and versions to be about $1-1.5$ (on a scale of $0-4$ ), which represents between 0.5 and $0.75 \mathrm{~mm}$, respectively. However, these values can be lower when considering modern silicone hydrogel CL because these lenses previously were fitted tighter than older low-Dk lenses and therefore moved less. Indeed, more recently Wolffsohn et al. [38] used video recordings to observe that the version lags of soft contact lenses might be an average of about $0.5 \mathrm{~mm}$.

The Acuvue Oasys lens for presbyopia showed higher variability than the Biofinity D lens, which might be explained by the aspheric nature of the Biofinity D lens with smooth transition of power and the multiconcentric nature of the Acuvue Oasys lens for presbyopia with abrupt changes in power. In other words, while $0.5-\mathrm{mm}$ displacement in an aspheric lens might have less of an impact on the outcomes of peripheral refraction reading, this impact might be higher for lenses with rapid and/or alternating changes of power. The lens design differences also might explain the lack of changes in refraction across the eccentricities of the visual field compared with the center with the Acuvue Oasys lens for presbyopia. The 
diameter of the measurement area with the Grand Seiko autorefractor/keratometer is larger than the size of the individual concentric rings of approximate uniform power. As a result, each single measurement might include information from two adjacent portions of the lens and produce an aliasing effect. Thus, the absence of changes in the peripheral refractive pattern across the visual field with the Acuvue Oasys lens for presbyopia might not be discarded, but also cannot be measured with this methodology.

As recently suggested, the center-distance design MFCL (Proclear D lens) can induce peripheral myopic defocus $[17,18]$ and the same behavior was verified in this work using two different measuring methods to determine peripheral refraction with an open-field autorefractor and aberrometer. The differences between values obtained by ocular and head rotation did not manifest significant difference both statistically and clinically. The analysis with MANOVA considered all factors that could influence refraction obtained across the horizontal visual field. But as many factors were put together in same test and under same statistical analysis this could minimize the importance/significance of the differences and for this reason some substantial differences that could be seen in the charts did not archieve statistical significance. However, regarding research and particularly if we attempt to compare the outcomes of different lens designs and fitting strategies, the influence of each measuring method on peripheral refraction should be considered carefully. Measuring method by head rotation was performed in previous studies using contact lenses $[27,28]$ to limit potential translocation of the contact lenses associated with large eye turn. However, considering the current data, refraction measured with eye rotation may provide results which are comparable with head rotation in eyes fitted with a MFCL. Ticak and Walline [29] did not obtain peripheral myopic defocus in patients wearing Proclear D lenses, which is very unlikely considering the previous research. A limitation of our results is that we represent the amount of change that is being induced. However this does not consider the actual position of the peripheral foci regarding the retina. Such information would be essential in clinical trials where the potential effect of peripheral defocus might be relevant to interpret the outcomes in terms of myopia progression. But the purpose of our study is to evaluate the change induced by the lenses as measured with either method (eye rotation and head rotation). The authors argued that lens movement on eye rotation might be responsible for this. In our previous studies [17,19] and in a study of Rosén et al. [18] a significant change toward higher peripheral myopic defocus was found with the Proclear D lens. In the current work, the Biofinity D lens showed a trend toward induction of peripheral myopic defocus of about $-1.00 \mathrm{D}$ at $30^{\circ}$ of eccentricity. The fact that Ticak and Walline did not find similar results, but rather an opposite trend with peripheral relative hyperopia, might be explained by a different dynamic behavior of the lens on peripheral eye versions in their population or by differences in the methodology used to control ocular fixation Same amount of myopic shift may have been induced in the current study as by Ticak and Walline [29], but if baseline data is not the same, this could be a possible explication for different effect of these MFCL at relative PR in present work. By this reason we considered important represent present results as relative peripheral refractive error to can visualize the real shift of refractive error at each peripheral location respectively to baseline. However, our results do not support this hypothesis as our results for a lens with a similar optical design was not different rotating the head where potential lens displacement on lateral versions is not present. However, their measures showed large standard deviation on the peripheral measurements which suggests a heterogeneous behavior of the lens centration or the effect of the +2.00 add in different subjects; both factors could justify their results against the expected according to present study and previous research mentioned above. Additional justifications might be found on the different polymers used in Proclear D (Omafilcon A) and Biofinity $\mathrm{D}$ (Comfilcon A) with different modulus and different potential fitting behavior. Furthermore, in their study, Ticak and Walline used a +2.00 Add while we used a +2.50 . The current study cannot ensure that the same results could be obtained if applied to other lens material of different modulus, front and back surface designs, or even for lenses with the same design and material using different add powers.

Non-cycloplegic evaluation of axial and peripheral refractive errors might eventually be considered a limitation of the current study once refractive error could vary with accommodative demand. However, we guaranteed at least a distance of $2.5 \mathrm{~m}$ between the corneal apex and the fixation targets, which might minimize the effects of accommodation on our refractive error measurements. Another limitation must be considered: we only measured refraction along the horizontal visual field, which means that other future works has needed to evaluate the refractive pattern at vertical visual field.

In summary, we did not find differences in peripheral refraction measurements between eye rotation and head rotation methods using the Grand Seiko autorefractor/keratometer in eyes wearing two different MFCLs. The differences did not reach any statistical or clinical significance. The Grand-Seiko autorefractor seems to not be sufficiently sensitive to detect the changes between distance and near power areas with the Acuvue Oasys lens for presbyopia. Despite the fact that we could not detect a relative peripheral myopic defocus generated by the near vision zones across the horizontal visual field relatively to the center, we cannot eliminate the possibility that this lens changes the pattern of the peripheral refractive error.

\section{Acknowledgements}

The authors thank the companies that donated the contact lenses used in the study. The authors declare that they do not have any proprietary or financial interests in any of the materials mentioned in this article. This work was funded in part by FEDER through the COMPETE Program and by the Portuguese Foundation for Science and Technology (FCT) in the framework of projects PTDC/SAU-BEB/098391/2008, PTDC/SAU-BEB/098392/2008 and the Strategic Project PEST-C/FIS/UI607/2011. This study has been presented at American Academy of Optometry, Seattle 2013, October 24th to 27 th.

\section{References}

[1] Charman WN, Radhakrishnan H. Peripheral refraction and the development of refractive error: a review. Ophthalmic Physiol Opt 2010;30(4):321-38.

[2] Wallman J, Winawer J. Homeostasis of eye growth and the question of myopia. Neuron 2004;43:447-68.

[3] Atchison DA, Pritchard N, Schmid KL. Peripheral refraction along the horizontal and vertical visual fields in myopia. Vision Res 2006;46(8-9):1450-8.

[4] Faria-Ribeiro M, Queirós A, Lopes-Ferreira D, Jorge J, González-Méijome JM. Peripheral refraction and retinal contour in stable and progressive myopia. Optom Vis Sci 2013;90(1):9-15.

[5] Smith III EL, Hung L-F, Huang J. Relative peripheral hyperopic defocus alters central refractive development in infant monkeys. Vision Res 2009;49(19):2386-92.

[6] Bakaraju RC, Ehrmann K, Ho A, Papas EB. Pantoscopic tilt in spectaclecorrected myopia and its effect on peripheral refraction. Ophthalmic Physiol Opt 2008;28(6):538-49.

[7] Tabernero J, Vazquez D, Seidemann A, Uttenweiler D, Schaeffel F. Effects of myopic spectacle correction and radial refractive gradient spectacles on peripheral refraction. Vision Res 2009;49:2176-86.

[8] Lin Z, Martinez A, Chen X, Li L, Sankaridurg P, Holden BA, et al. Peripheral defocus with single-vision spectacle lenses in myopic children. Optom Vis Sci 2010;87(1):4-9.

[9] Cho P, Cheung SW, Edwards M. The longitudinal orthokeratology research in children (LORIC) in Hong Kong: a pilot study on refractive changes and myopic control. Curr Eye Res 2005;30(1):71-80. 
[10] Hiraoka T, Kakita T, Okamoto F, Takahashi H, Oshika T. Long-term effect of overnight orthokeratology on axial length elongation in childhood myopia: a 5-year follow-up study. Invest Ophthalmol Vis Sci 2012;53(7):3913-9.

[11] Walline JJ, Jones LA, Sinnott LT. Corneal reshaping and myopia progression. Br J Ophthalmol 2009;93(9):1181-5.

[12] Santodomingo-Rubido J, Villa-Collar C, Gilmartin B, Gutiérrez-Ortega R. Myopia control with orthokeratology contact lenses in Spain (MCOS): study design and general baseline characteristics. J Optom 2009;2(4):215-22.

[13] Cho P, Cheung SW. Retardation of myopia in orthokeratology (ROMIO) study: a 2-year randomized clinical trial. Invest Ophthalmol Vis Sci 2012:53(11):7077-85.

[14] Kakita T, Hiraoka T, Oshika T. Influence of overnight orthokeratology on axial elongation in childhood myopia. Invest Ophthalmol Vis Sci 2011;52(5):2170-4.

[15] Queirós A, Gonzalez-Meijome JM, Jorge J, Villa-Collar C, Gutiérrez AR. Peripheral refraction in myopic patients after orthokeratology. Optom Vis Sci 2010;87(5):323-9.

[16] Sankaridurg P, Holden B, Smith 3rd E, Naduvilath T, Chen X, de la Jara PL, et al. Decrease in rate of myopia progression with a contact lens designed to reduce relative peripheral hyperopia: one-year results. Invest Ophthalmol Vis Sci 2011;52(13):9362-7.

[17] Lopes-Ferreira D, Ribeiro C, Maia R, García-Porta N, Queirós A, Villa-Collar C, et al. Peripheral myopization using a dominant design multifocal contact lens. J Optom 2011;4(1):14-21.

[18] Rosén R, Jaeken B, Lindskoog Petterson A, Artal P, Unsbo P, et al. Evaluating the peripheral optical effect of multifocal contact lenses. Ophthalmic Physiol Opt 2012;32(6):527-34.

[19] Lopes-Ferreira D, Ribeiro C, Neves H, Faria-Ribeiro M, Queirós A, Villa-Collar C, et al. Effect of dominant design multifocal contact lenses on peripheral refraction in young myopic eyes. J Optom 2013;6:85-94.

[20] Walline JJ, Mcvey L. Myopia control with a soft bifocal contact lens (abstract) in myopia: proceedings of the 13th international conference. Optom Vis Sci 2010;88(3):395-403.

[21] Aller TA, Wildsoet C. Bifocal soft contact lenses as a possible myopia control treatment: a case report involving identical twins. Clin Exp Ophthalmol 2008;91(4):394-9.

[22] Aller TA, Laure A, Wildsoet C. Results of a one-year prospective clinical trial (CONTROL) of the use of bifocal soft contact lenses to control myopia progression. Ophthalmic Physiol Opt 2006;26(S1):8-9.

[23] Anstice NS, Phillips JR. Effect of dual-focus soft contact lens wear on axial myopia progression in children. Ophthalmology 2011;118(6):1152-61.
[24] Kollbaum PS, Jansen ME, Rickert ME. Comparison of patient-reported visua outcome methods to quantify the perceptual effects of defocus. Contact Lens Anterior Eye 2012;35(5):213-21.

[25] Seidemann A, Schaeffel F, Guirao A, Lopez-Gil N, Artal P. Peripheral refractive errors in myopic, emmetropic, and hyperopic young subjects. J Opt Soc Am (A) 2002;19(12):2363-73.

[26] Radhakrishnan H, Charman WN. Peripheral refraction measurement: does it matter if one turns the eye or the head? Ophthalmic Physiol Opt 2008;28(1):73-82

[27] Shen J, Clark CA, Soni PS, Thibos LN. Peripheral refraction with and without contact lens correction. Optom Vis Sci 2010;87(9):642-55.

[28] Kwok E, Patel B, Backhouse S, Phillips JR. Peripheral refraction in high myopia with spherical soft contact lenses. Optom Vis Sci 2012;89(3):263-70.

[29] Ticak A, Walline JJ. Peripheral optics with bifocal soft and corneal reshaping contact lenses. Optom Vis Sci 2013;90(1):3-8.

[30] Kang P, Fan Y, Oh K, Trac K, Zhang F, Swarbrick H. Effect of single vision soft contact lenses on peripheral refraction. Optom Vis Sci 2012;89(7): 1014-21.

[31] Queirós A, Jorge J, González-Méijome JM. Influence of fogging lenses and cycloplegia on peripheral refraction. J Optom 2009;2(2):83-9.

[32] Queirós A, González-Méijome J, Jorge J. Influence of fogging lenses and cycloplegia on open-field automatic refraction. Ophthalmic Physiol Opt 2008;28(4):387-92.

[33] Mathur A, Atchison DA, Kasthurirangan S, et al. The influence of oblique viewing on axial and peripheral refraction for emmetropes and myopes. Ophthalmic Physiol Opt 2009;29(2):155-61.

[34] Harris WF. Dioptric power: its nature and its representation in three- and fourdimensional space. Optom Vis Sci 1997:74(6):349-66.

[35] Thibos LN, Wheeler W, Horner D. Power vectors: an application of Fourier analysis to the description and statistical analysis of refractive error. Optom Vis Sci 1997;74(6):367-75.

[36] Macfadden LA, Gray LS, Strang NC, Seidel D. The effect of eye rotation on measurements of peripheral retinal shape using the IOLMaster. Invest Ophthalmol Vis Sci 2007; 48 (E-Abstract 4002).

[37] Young G, Holden B, Cooke G. Influence of soft contact lens design on clinical performance. Optom Vis Sci 1993;70(5):394-403.

[38] Wolffsohn JS, Hunt Oa, Basra AK. Simplified recording of soft contact lens fit. Contact Lens Anterior Eye 2009;32(1):37-42 